\begin{tabular}{|c|c|}
\hline \multirow{3}{*}{ 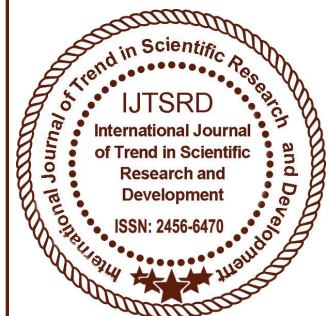 } & $\begin{array}{l}\text { International Journal of Trend in Scientific } \\
\text { Research and Development (IJTSRD) }\end{array}$ \\
\hline & International Open Access Journal \\
\hline & ISSN No: 2456 - 6470 | www.ijtsrd.com | Volume - 1 | Issue - 5 \\
\hline
\end{tabular}

\title{
Comparative analysis of AODV and DSDV routing protocols for Mobile Ad Hoc Networks
}

\author{
Anurag Misra \\ Training Specialist, HIPF, Riyadh
}

\begin{abstract}
Mobile Ad hoc Networks (MANETs) are collection of wireless mobile nodes. MANETs are infrastructure less temporary networks. They do not have fixed static infrastructure. As no fixed infrastructure is available all nodes itself, work as receiver, transmitter as well as router. These nodes are also mobile so providing stable route is a big challenge. We have different approaches to find route in MANETs and all approaches use some protocols. This paper concentrates upon the analysis of two Mobile Ad Hoc Networking protocols. Ad hoc On demand DistanceVector (AODV) and Destination-Sequenced DistanceVector (DSDV). DSDV uses proactive or table driven approach to route the data whereas AODV is a reactive protocol and uses on demand routing. Main target of this paper is to highlight different aspect of both protocols and compare them. So, one can decide which protocol is better suited to their needs.
\end{abstract}

\section{INTRODUCTION}

Mobile Ad hoc Network (MANET) is a collection of nodes which all can communicate without any fixed infrastructure, over a wireless medium. All the nodes work as hosts as well as router. And all of them are mobile. Nodes can directly communicate with each other or they can use other intermediate nodes to forward data from source to destination. In MANETs almost everything is changeable including number of nodes, speed, topology etc. As MANETs are made up of independent nodes and nothing is fixed in MANETs. So, networks have to be self configurable. With so many constraints like limited bandwidth, speed and energy developing protocols for MANETs is a great challenge. There are three main approaches to develop routing protocols for MANETs and those are shown below in figure 1.1 .

Keywords: MANET, Protocol, DSDV, AODV, Proactive, Reactive, Routing, Route discovery

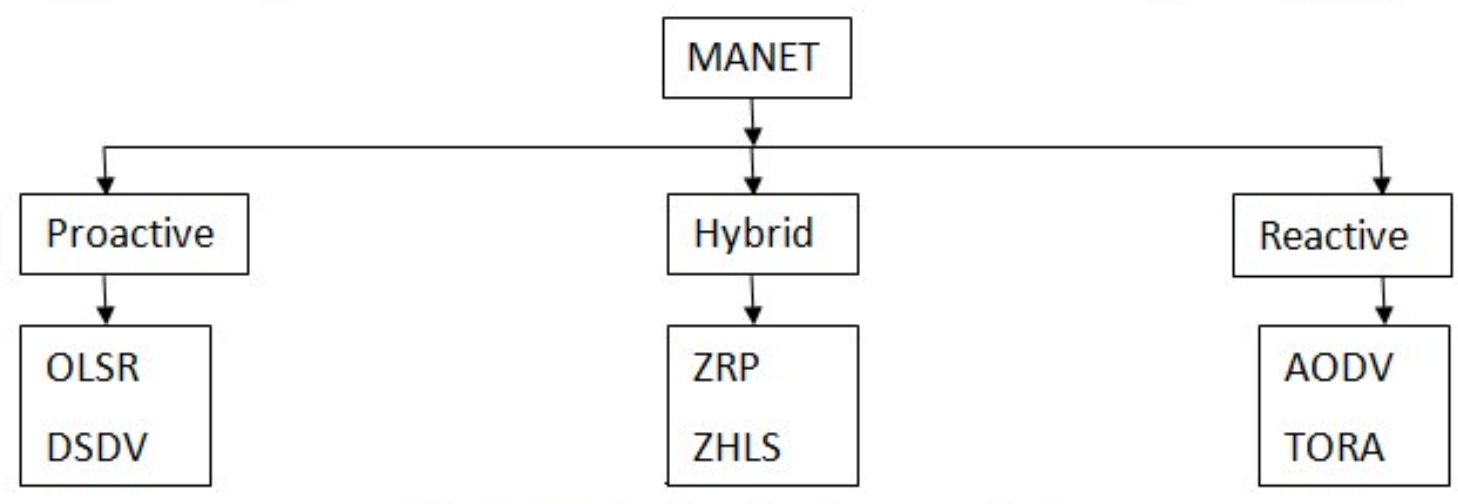

Figure 1.1 


\section{Proactive (Table Driven) Routing Protocol}

In proactive routing protocols each node maintains a routing table just like we have in general wired routing protocols. That's why these protocols are called table driven protocols. Table contains latest information about routes and cost of routes to reach a particular destination. When any change in the topology occurs or any new node is added route update information is sent to all the nodes by broadcasting. These protocols are not able to perform well if network topology changes very frequently or if network has too many nodes because of too much routing overheads. Some of the proactive protocols are Destination Sequenced Distance Vector (DSDV) and Optimized Link State Routing (OLSR). In this paper our main concern will be DSDV for our study.

\subsection{Destination Sequenced Distance Vector (DSDV)}

Destination Sequenced Distance Vector (DSDV) is proactive table driven protocol. It is based on Bellman-Ford algorithm. DSDV is developed by C. Perkins and P. Bhagwat in 1994. In DSDV, each node maintains a routing table which contains all required destinations within the network and the number of hops needed to reach each destination. Loop formation problem of Bellman-Ford algorithm is solved in DSDV by marking each entry of routing table with a sequence number. The route labeled with a highest sequence number is used to send data.

Two types of route update techniques are used: full dump update and incremental update. Full dump update sends the complete routing table and its data could span to many packets. Incremental update sends only those entries which all have changed metrics since the last update and it must fit in single packet. If there is some space left in that packet then it also includes entries where sequence number is changed. When we have a stable network incremental updates are used to avoid extra traffic. Loop free paths are guaranteed in DSDV protocol. On receiving an update packet, DSDV updates routing table if one of the following conditions are satisfied:

a) $\mathrm{Sn}>\mathrm{Se}$ or

b) $\mathrm{Sn}=\mathrm{Se}$ otherwise update packet is ignored.

Here, $\mathrm{Sn}$ and Se are the sequence numbers of new message and existing message respectively.

\section{Reactive (On Demand) Routing Protocol}

Reactive Routing Protocols don't keep permanent routing tables and don't react on any changes in topology or on addition of a new node. These protocols search for a route only when some node attempts to send data over the network. When a node attempts to send data, it request for a route and then only these protocols searches for a route by flooding the route request packets throughout the network. Some of the reactive routing protocols are Ad hoc On demand Distance Vector (AODV) and Temporarily Ordered Routing Algorithm (TORA). In this paper our main concern will be AODV for our study.

\subsection{Ad hoc On demand Distance Vector (AODV)}

Ad hoc On demand Distance Vector is a reactive on demand routing protocol for MANETs. In AODV protocol nodes don't maintain the routing table all the time, they don't need to check constantly for any topology change in network. Here nodes remain silent; they don't bother about routes, topology changes or about any node being added or removed, until a node wants to send data over the network. Routes are searched and used only at the time of need and only for as long as they are needed. AODV support unicast and multicast messages also. To support multicast, AODV forms trees which are composed of group members of multicast. Like DSDV, AODV also uses sequence number to avoid loops and to ensure the freshness of the route. AODV is self-starting protocol and it's scalable to quite large number of nodes. When a node wants to transfer data over the network, that source node broadcasts route request (RREQ). On receiving of this broadcast all other nodes check if it is destined to them or if they have route entry for the destination. If both the conditions are not satisfied then they forward it and record the address of node from which they received the request. An explosion of temporary routes is created. If request receiving node finds out that it's destined for itself or it has a route entry to destination it sends route reply (RREP) message using one of the temporary routes. Source node receives all possible routes and uses the route having least hop count. Unused nodes are discarded after timeout. Selected route is stored till transmission is complete. But if in middle of transmission source receives another route reply (RREP) message with greater or same sequence number but smaller hop count, it may update its routing information and can switch to new route for remaining transmission. A route remains active as long as data packets are transferred periodically from 
International Journal of Trend in Scientific Research and Development (IJTSRD) ISSN: 2456-6470 source to destination using that route. If no data packet is transferred till time out reached, route information is deleted from routing table of all intermediate nodes. If any failure occurs during transmission route error (RERR) message is sent to source, to inform the source that destination is unreachable. Conceptually, in AODV route error

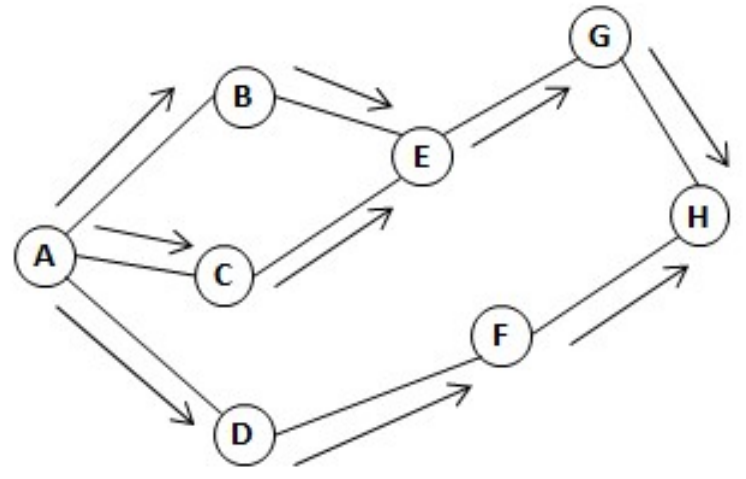

I) Propagation of Route Request (RREQ) message propagation can be visualized as a tree with node at point of error as root and all source nodes using failed link as leaves.

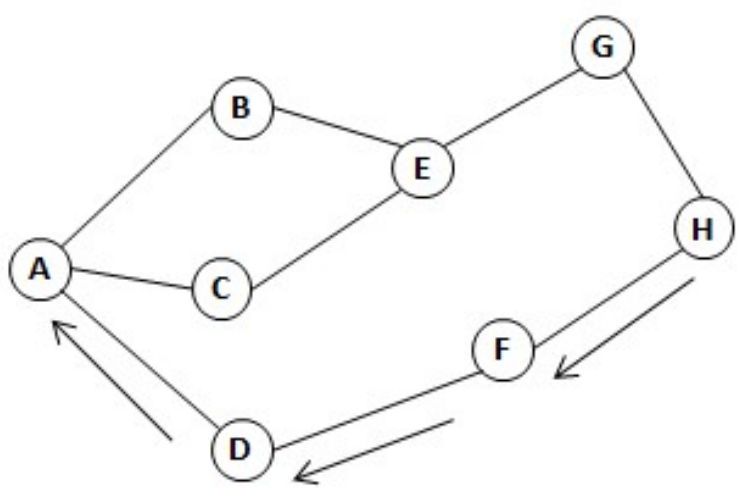

II) Propagation of Route Request (RREP)

4. Table 1 - Shows Comparison of DSDV and AODV based on certain Protocol Properties, features and techniques used by protocol:

\begin{tabular}{|l|l|l|l|}
\hline S. No. & \multicolumn{1}{|c|}{ Protocol Property } & \multicolumn{2}{c|}{ DSDV } \\
\hline $\mathbf{1}$ & Table Driven / Source Routing & Table Driven & $\begin{array}{l}\text { Table Driven with Source } \\
\text { Routing }\end{array}$ \\
\hline $\mathbf{2}$ & Need of Hello Message & Yes & Yes \\
\hline $\mathbf{3}$ & Route Selection & Link State & Shortest and Updated Path \\
\hline $\mathbf{4}$ & Route Update & Periodic & Non periodic \\
\hline $\mathbf{5}$ & Route Computation Update & Distributed & Broadcast \\
\hline $\mathbf{6}$ & Route Mechanism / Maintenance in & Route table with next hop & Route table with next hop \\
\hline $\mathbf{7}$ & Method & Broadcast & Unicast \\
\hline $\mathbf{8}$ & Network Overheads & High & Medium \\
\hline $\mathbf{9}$ & Node / Caching Overheads & Medium & Low \\
\hline $\mathbf{1 0}$ & Routing Overheads & Medium & High \\
\hline $\mathbf{1 1}$ & Update Information & Distance Vector & Route Error \\
\hline $\mathbf{1 2}$ & Loop Free & Yes & Yes \\
\hline $\mathbf{1 3}$ & Route Discovery & No & Yes \\
\hline $\mathbf{1 4}$ & Route Maintenance & No & Yes \\
\hline $\mathbf{1 5}$ & Multi Hop Wireless Support & Yes & Yes \\
\hline $\mathbf{1 6}$ & Multiple Routes & No & No \\
\hline $\mathbf{1 7}$ & Unidirectional Link Support & No & No \\
\hline $\mathbf{1 8}$ & Number of nodes Suitability & Less number of nodes & Highly dynamic \\
\hline $\mathbf{1 9}$ & Protocol Type & Proactive & Reactive \\
\hline $\mathbf{2 0}$ & Packet Size & Uniform & Uniform \\
\hline $\mathbf{2 1}$ & Routing Philosophy & Flat & Flat \\
\hline $\mathbf{2 2}$ & Multicast Support & No & Yes \\
\hline $\mathbf{2 3}$ & Distributed & Yes & Yes \\
\hline $\mathbf{2 4}$ & Periodic Broadcast & Yes & No \\
\hline $\mathbf{2 5}$ & Quality of Service Support & No & No \\
\hline
\end{tabular}


International Journal of Trend in Scientific Research and Development (IJTSRD) ISSN: 2456-6470

Table 1 shows the comparison of both of the protocols. But this comparison is still quite theoretical. Although it's such a descriptive and elaborate theoretical comparison that, one can easily decide which type of protocol from AODV and DSDV is best suited to their needs but still its good idea to come up with a firm practical data to compare the actual performance of both the protocols. That can be quite ensuring than just the comparative theoretical analysis. That's why now we will have some simulated data for both of the protocol to show how they perform in simulated conditions and parameters.

\section{Random Way Point model}

Random Way Point model is used for this simulation. This model is first proposed by Johnson and Maltz. And it became a benchmark to evaluate MANET routing protocols due to its simplicity and wide availability. RWP model is a random model for mobile users and how their location, acceleration and velocity change over time. In RWP model initially, all the nodes are distributed randomly over the simulation area. Node selects its random destination among the other nodes with in simulation area. After selection of this random destination, velocity is chosen from a uniform distribution. After reaching destination node, a small "pause time" is given and then again next random destination is selected by $\mathrm{MN}$. $\mathrm{MN}$ also chooses a speed uniformly distributed between minimum to maximum speed. To reflect the degree of mobility some parameters could be set for value of travelling speed which is chosen uniformly in the interval. At this selected speed, $\mathrm{MN}$ continues its journey towards newly selected destination. After reaching on destination again the whole process is repeated after pause time.

\section{Simulation analysis and results}

For this study topology of fixed area of $700 \times 700 \mathrm{~m}^{2}$ is used with varying number of nodes $25,50,75,100$. Speed used is $20 \pm 3 \mathrm{~m} / \mathrm{s}$, pause time is $15 \pm 3 \mathrm{~s}$, packet size is $512 \mathrm{~B}$, simulation time is $300 \mathrm{~s}$ and traffic node is $10,20,40,60$ respectively with 25,50 , 75,100 nodes in simulation. We consider effect of mobility on 4 major factors. These are Packet Delivery Ratio, Average End to End Delay, Normalized Routing Overheads and Throughput of the MANET.

\subsection{Packet Delivery Ratio}

Packet Delivery Ratio is the ratio of total number of packets successfully received by the destination nodes to the number of packets sent by the source nodes throughout the simulation. It also describes the loss rate of the packets, which affects the maximum throughput that can be supported by the network. Formula to calculate Packet Delivery Ratio is as follows:

$\mathrm{PDR}=(\mathrm{Pr} / \mathrm{Ps}) * 100$

Where $\quad \mathrm{PDR}=$ Packet Delivery Ratio

$\operatorname{Pr}=$ Total number of packets received

Ps $=$ Total number of packets sent 


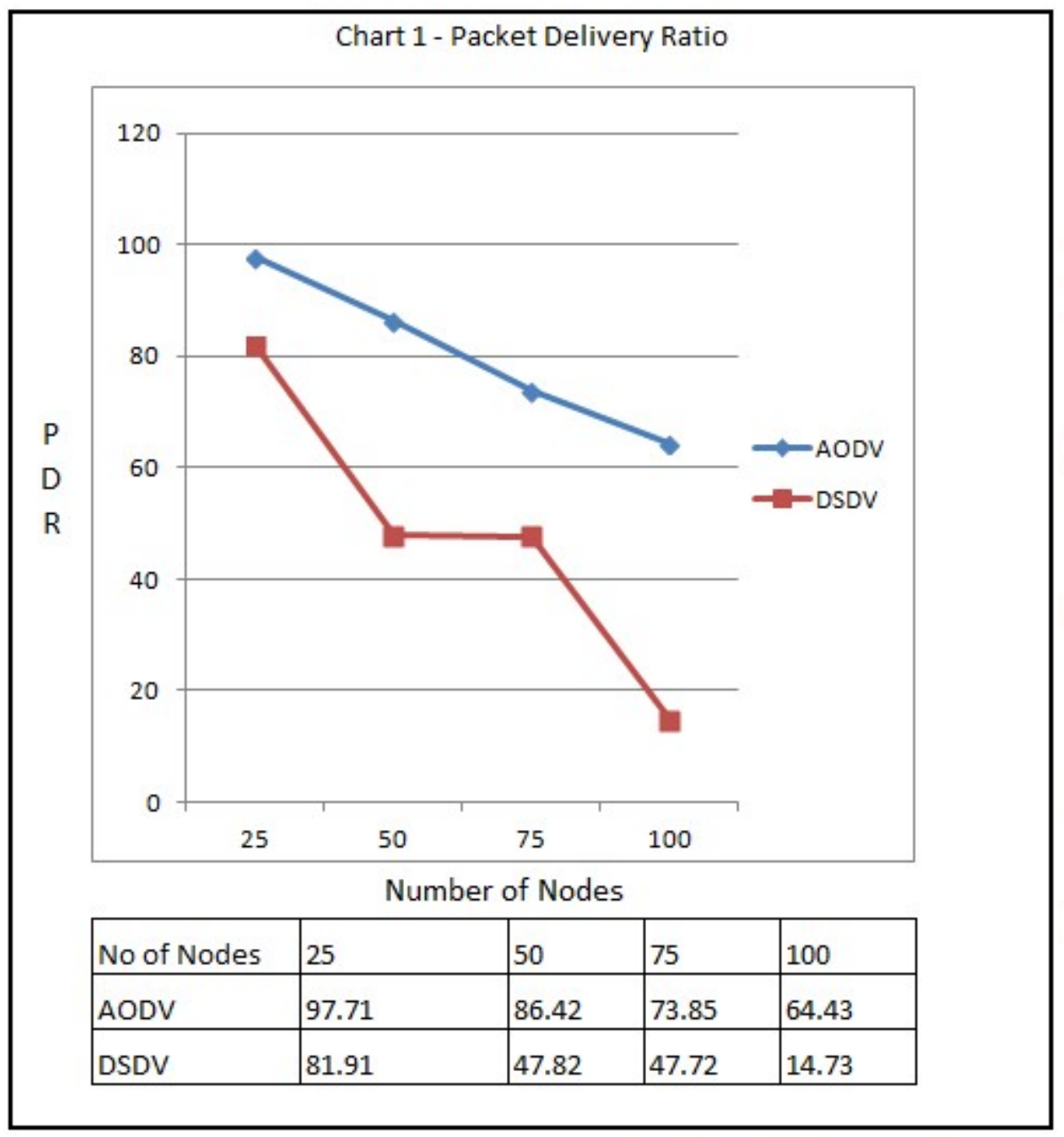

\subsection{Average End to End Delay}

Average End to End Delay is defined as the average delay in the transmission of a packet between both nodes. A higher value of end to end delay means that the network is congested and the routing protocol does not perform well. Average end to end delay is calculated using following formula:

$\mathrm{AD}=\Sigma(\mathrm{Ta}-\mathrm{Ts}) / \mathrm{n}$

Where $\quad \mathrm{AD}=$ Average Delay

$\mathrm{Ta}=$ Arrival time of packet

Ts $=$ Start time of packets 


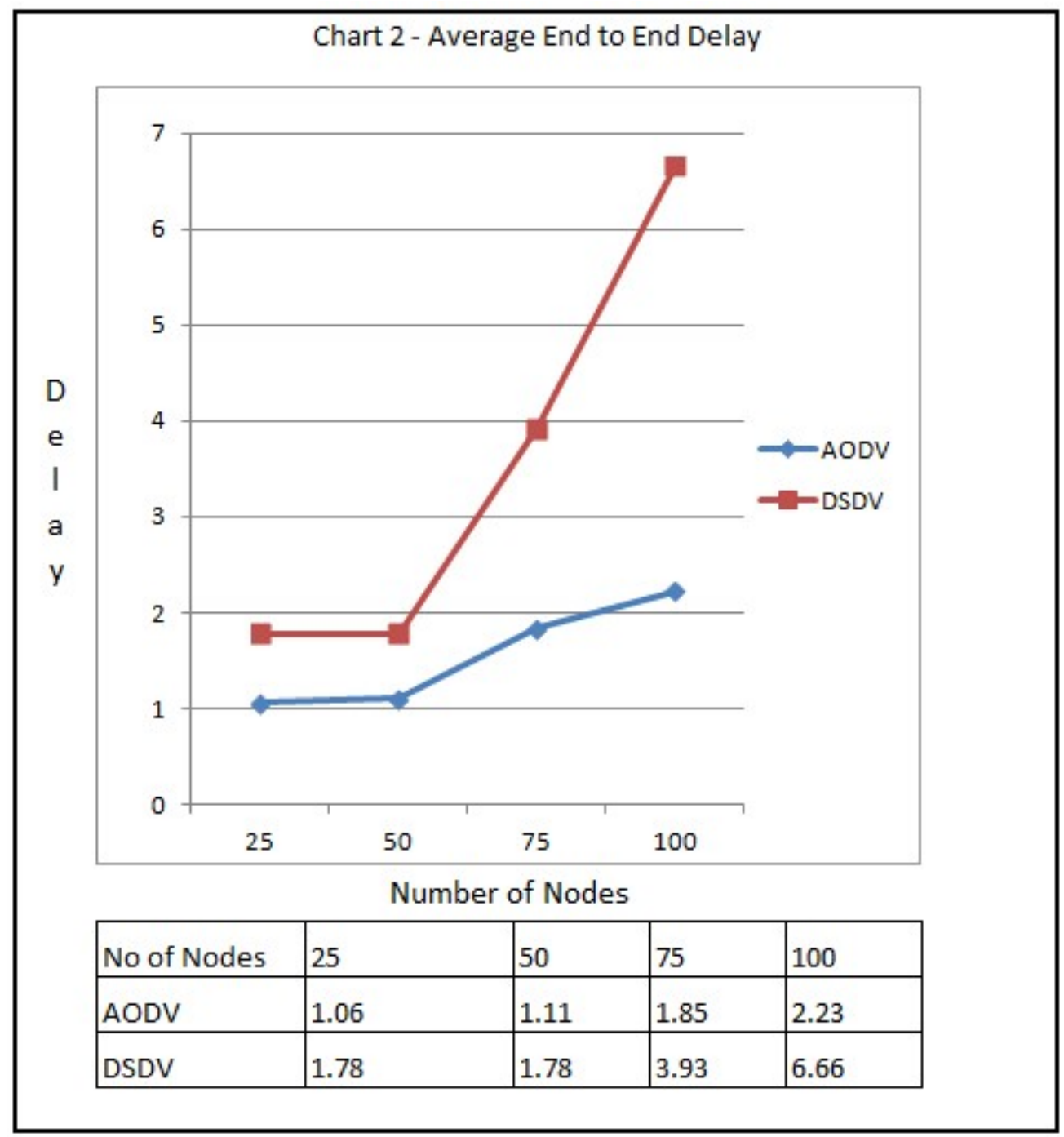

\subsection{Normalized Routing Load}

Normalized Routing Load is calculated as the ratio between the numbers of routing packets transmitted to the number of packets actually received means it's an accounting of dropped packets. The higher NRL value means higher overheads of routing packets and consequently lower the efficiency of the protocol. NRL is defined as number of routing packets transmitted per data packets delivered at destination. Each hop wise transmission of a routing is counted as one transmission. It is sum of all control packets sent by all nodes in network to discover and maintain route. The formula by which Normalized Routing Load is calculated is:

$\begin{array}{ll}\text { Where } & \text { NRL Pro } / \text { Pre } \\ & \text { NRL }=\text { Normalized Routing Load } \\ & \text { Pro }=\text { Routing Packets } \\ & \text { Pre }=\text { Received Packets }\end{array}$ 
International Journal of Trend in Scientific Research and Development (IJTSRD) ISSN: 2456-6470

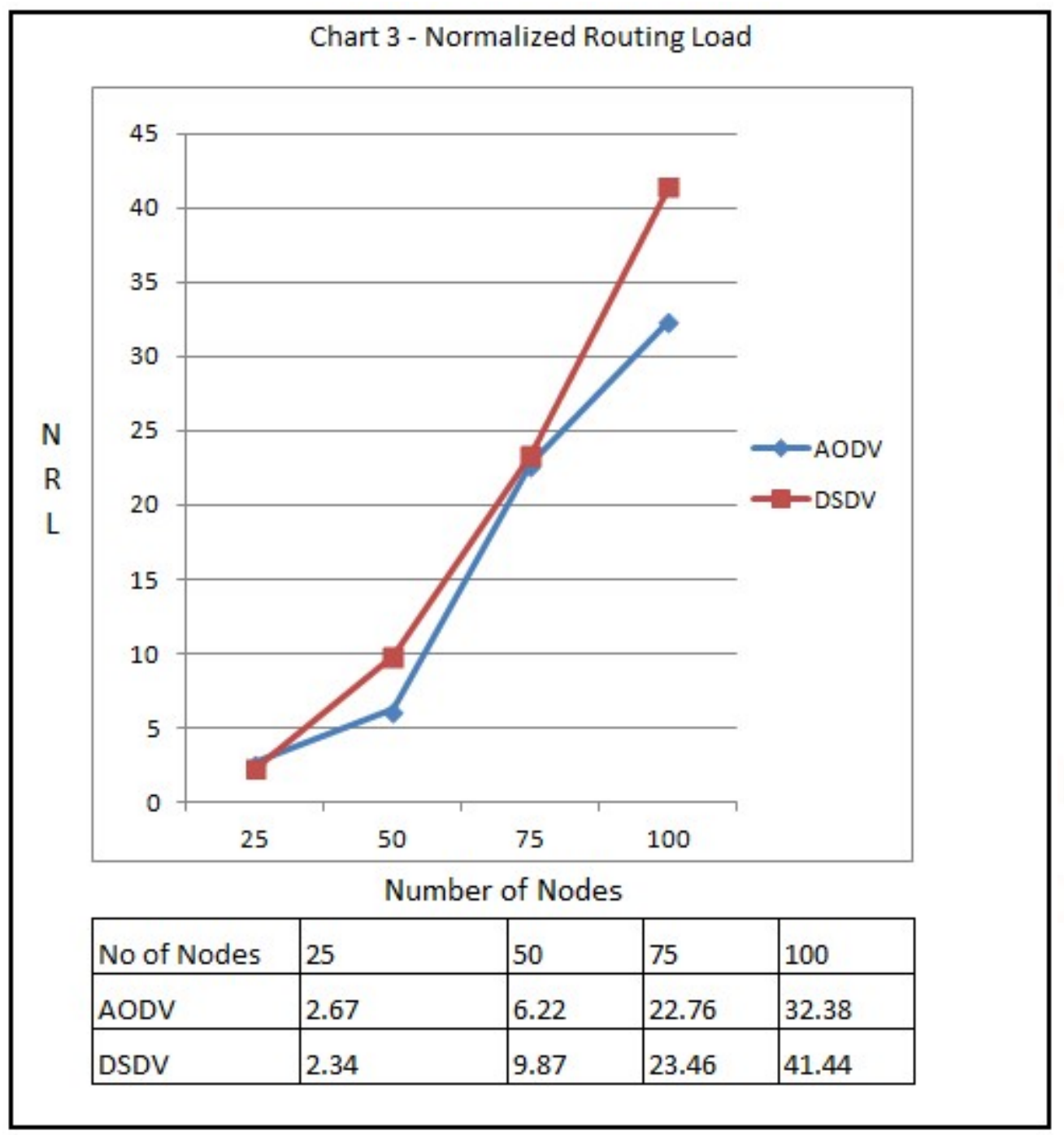

\subsection{Throughput}

Throughput is the average rate at which the total number of data packets are delivered successfully from one node to another over a network. Measuring unit for throughput is $\mathrm{KB} / \mathrm{Sec}$ and formula by which throughput is calculated is as follows:

$$
\mathrm{Tp}=(\mathrm{NPD} * \mathrm{Ps}) / \mathrm{TDS}
$$

Where $\quad T p=$ Throughput

$\mathrm{NPD}=$ Number of delivered packets

Ps $=$ Packet Size

TDS $=$ Total duration of simulation 


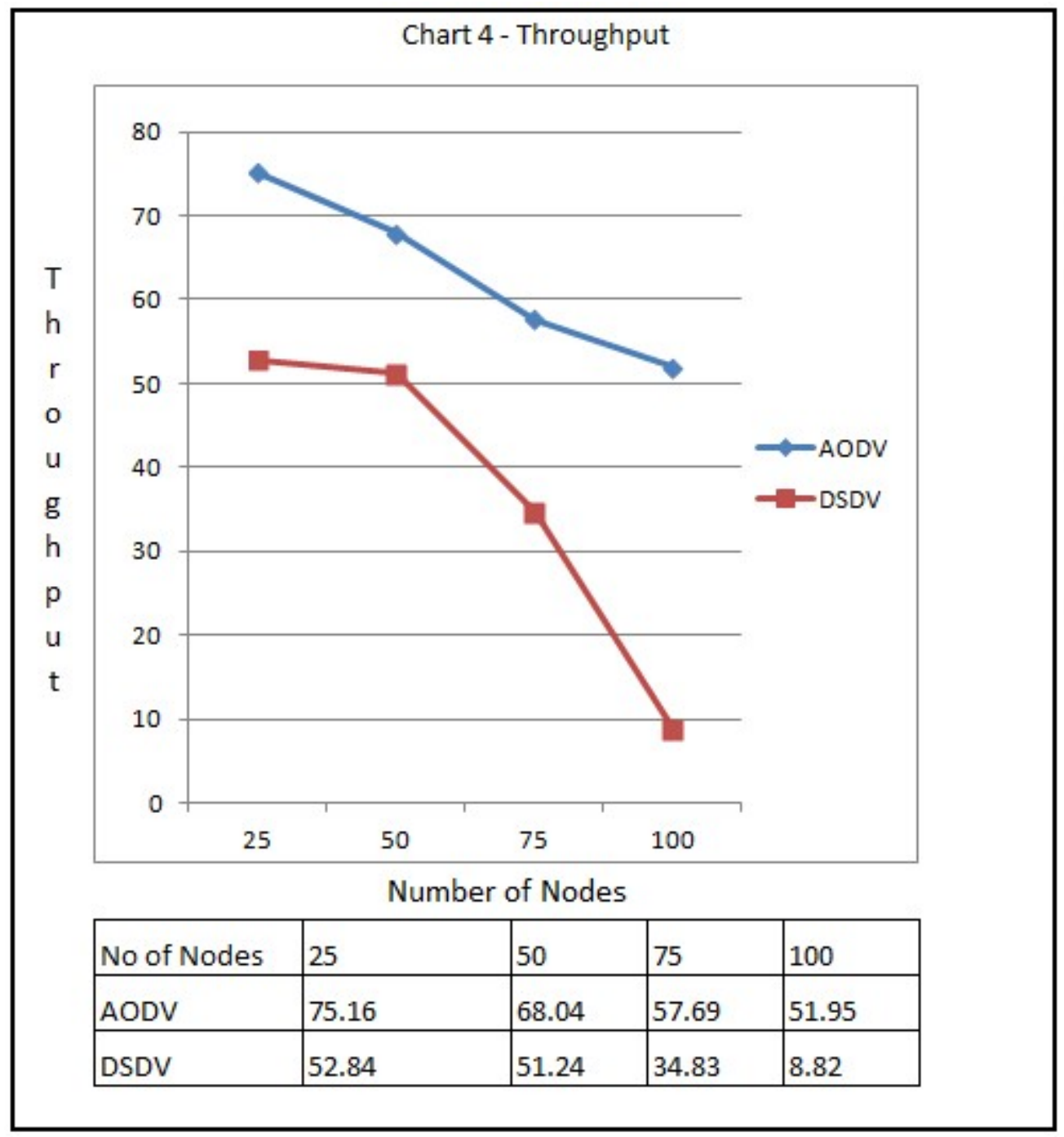

\section{Conclusion}

In this paper, we have tried to analyze two routing protocols which both are used for MANETs. And both of them are using different approaches for routing. DSDV is a proactive table driven protocol whereas AODV is reactive on demand protocol. We have compared both of them for Packet Delivery Ratio, Average End to End Delay, Normalized Routing Load and Throughput. Random Waypoint model is used for the simulation by which this data is collected. As per results it is very clear that increasing number of nodes affects both the protocols to a very great extent. But still it affects DSDV more than AODV. DSDV is not bad for lesser number of nodes but when number of nodes increase from 100 or above performance of DSDV decreases drastically. But although AODV performance also decreases when number of nodes are increased but still it performs much better. Only for Normalized Routing Load DSDV's and AODV's performance is quite comparable. But for Packet Delivery Ratio, Average end to end delay and Throughput, AODV clearly outperforms DSDV, especially for greater number of nodes.

\section{References}

[1] C. E. Perkins and P. Bhagwat, "Highly Dynamic Destination Sequenced Distance-vector Routing (DSDV) for Mobile Computers", Proceedings of the ACM SIGCOMM 94 Conference, August 1994, pages 234-244.

[2] Sunil Kr. Kaushik, Sukhbir Singh, Kavita Chahal and Sandeep Dhariwal, "Performance Evaluation of Mobile Ad Hoc Networks with Reactive and Proactive Routing Protocols and Mobility Models", International Journal of Computer Applications (0975-8887) Volume 54-No.17, September 2012 
International Journal of Trend in Scientific Research and Development (IJTSRD) ISSN: 2456-6470

[3] Basudev Shivahare, Charu Wahi and Shalini Shivhare, "Comparison of Proactive and Reactive Routing Protocols in Mobile Ad hoc Network using Routing Protocol Property", International Journal of Emerging Technology and Advanced Engineering, ISSN 2250-2459, Volume 2, Issue 3, March 2012.

[4] Prabhjot Kaur, Dr. Shaveta Rani and Oaramjeet Singh, "International Journal of Advanced Research in Computer Science and Software Engineering”, Volume 5, Issue 4, April 2015

[5] Sachin Kumar Gupta and R.K. Saket, "Performance Metric Comparison of AODV and DSDV Routing Protocols in MANETs using NS-2”, IJRRAS, Volume 7, Issue 3

[6] P. Manickam, T. Guru Baskar, M. Girija, and Dr. D. Manimegakai, "Performance Comparison of Routing Protocols in Mobile Ad hoc Networks", International Journal of Wireless \& Mobile Networks, Volume 3, No. 1, February 2011

[7] S.A. Ade and P.A. Tijare, "Performance Comparison of AODV, DSDV, OLSR and DSR Routing Protocols in Mobile Adhoc Networks", International Journal of Information Technology \& Knowledge Management

[8] S. Mohapatra and P. Kanungo, "Performance analysis of AODV, DSR, OLSR and DSDV Routing Protocols using NS2 Simulator”, SciVerse ScienceDirect, Procedia Engineering 30 (2012) 69 - 76

[9] V.K. Taksande and Dr. K.D. Kulat, "Performance Comparison of DSDV, DSR, AODV Protocol with IEEE 802.11 MAC for Chain Topology for Mobile Ad-hoc Network using NS-2", IJCA Special Issue on " 2 nd National Conference - Computing, Communication and Sensor Network" CCSN, 2011 